\title{
Elegance of science Coordinating an annual scientific art contest at the library
}

\author{
"After a certain high level of technical skill \\ is achieved, science and art tend to coalesce \\ in esthetics, plasticity, and form. The greatest \\ scientists are always artists as well."-Albert \\ Einstein
}

$\mathbf{T}$ Me Marston Science Library is the library on the University of Florida (UF) campus that supports the programs within the agricultural, life, engineering, physical, mathematical, and earth sciences. In fall 2008, the Marston Science Library held its first annual Elegance of Science art contest. The event was a huge success and culminated in the display of 116 pieces of artwork, including everything from mosaics collected from the surface of solid lubricant material to compounds detected using mass spectrometry imaging. The main goal of the contest was to increase the UF Libraries' overall mission to serve as places for contemplation and discovery.

\section{Proposal}

In 2008, Marston submitted a proposal to UF Libraries administration for contest funding. The proposal contained a justification that included information on the background, motivation, and overall goals of the contest and how these could clearly be linked to the UF Libraries' overall mission.

Additionally, it included a brief timeline, a proposed budget, publicity information, and contest rules, as well as information on receiving submissions, judging, and potential partners/sponsors for the contest. The following sections will discuss these components.

\section{Proposed budget}

Our budget for the contest was fairly straight forward. It itemized the funds required for publicity, printing, and dry mounting of images, cash prizes, and food for the awards reception. We wanted to offer cash prizes to the winners, in the hopes that it would increase the number of entries. We proposed these cash prizes would be awarded to the first- (\$150), second- (\$100), and third- (\$50) place winners. Providing cash prizes from the library's budget would have been problematic due to restrictions in how state funds can be spent. Fortunately this issue was bypassed through the offer of funds from an anonymous donor. In addition, the donor committed to supporting the contest's prize money for several subsequent years. We were allotted $\$ 160$ for reception food. As the event had a great turnout, both in the number of submissions and the reception attendance, the libraries have agreed to allot $\$ 300$ annually for the reception.

\section{Publicity}

In order for the Elegance of Science to be a success we needed to publicize both the contest and the reception. The first step was to create a Web site (www.uflib.ufl.edu/msl/ art) and a submission form. Postcards, posters, table tents, and news releases were then created. The postcards outlining entry guide-

Amy G. Buhler is assistant university librarian/ engineering librarian, e-mail: abuhler@ufl.edu, and Valrie Davis is assistant university librarian/engineering librarian, e-mail: vdavis@ufl.edu, at the University of Florida's Marston Science Library

๑ 2010 Amy G. Buhler and Valrie Davis 
lines were sent to targeted science, health science, and art departments on campus. In addition, postcards publicizing the reception were sent to these same departments. An e-mail was sent to respective library liaisons for dissemination to their faculty, staff, and students.

General publicity materials regarding the contest submission process were sent to the local city newspaper, campus newspaper, library newsletter, and various other campus media. Additional public relations vehicles included time on the university's electronic marquee and PowerPoint slides on televisions at the resident halls and at the various libraries, as well as links on various Web pages. Printed flyers were placed at the student union food court.

\section{Contest rules}

The contest rules were as follows:

- open only to current faculty, students, and staff of the University of Florida;

- artwork was limited to two-dimensional images;

- a maximum of five submissions per contestant;

- contestants had to submit images in JPEG format along with a description; and

- artwork had to be original and created by the contestant(s).

\section{Receiving and displaying submissions}

We decided to use the university's server to host a form that was created with the help of an outside programmer. All contestants were required to check a box accepting the rules and copyright statement. A Creative
Commons Attribution statement was used (creativecommons.org/licenses/by-nc-nd/3.0 /us/) to allow the libraries to display, preserve, and print the submissions.

Once the submission form was operational there were very few problems. The only exception was the few times that files were not received with the submission form, but since the inbox was monitored on a daily basis, we were able to quickly follow up with those entries missing attachments.

Image quality was an important aspect because printing required a high resolution, which subsequently meant large files. In an effort to not exceed inbox space, we decided to initially request low resolution JPEGs. The winning contestants were later asked to submit high-resolution JPEGs (600+ dpi) for printing purposes. Our public information officer put these images into an InDesign document and converted them to highquality PDFs (which is a vector image and can Mycorrhizas in Full Color-1st Place-Megan M. Smith, 2009, Soil and Water Science. "The hyphae and arbuscules of the mycorrhizal fungi that live in symbiosis with plant roots can be tricky to study under the microscope. After clearing and staining these sugar maple roots, the fungi pop into view in brilliant blue against the golden transparent root cells. This is an artistic rendering of the scene, acrylic on canvas." be enlarged better than a JPEG). Many of the images received were taken using high-end scientific equipment (i.e., electron microscope) and could be enlarged and printed without pixelation. In a couple of cases, printed images were slightly pixelated, which we attributed to images being taken with digital cameras below six megapixels or a lack of understanding regarding the adjustment of image resolution (i.e., contestant opened the image in Photoshop or a similar program and artificially changed the resolution).

It should be noted that you can not take a low resolution image and turn it into a 
high-resolution image simply by adjusting the dpi.

As we only had funds to print the winning entries, we wanted some way to showcase all the entries. We determined that an online slideshow (www.uflib.ufl.edu/msl/art /slideshowindex.html) would reach the broadest audience and turned to Google's Picasa for this purpose. We could also have this slideshow projected at the awards reception. As entries were received it was simple to transfer the images from the server directly into a Picasa album, along with the titles and descriptions of the works.

\section{Judging}

It was determined that a panel of judges would be solicited from the university community, specifically from the colleges related to the arts and sciences. To ensure balance, we had hoped to have

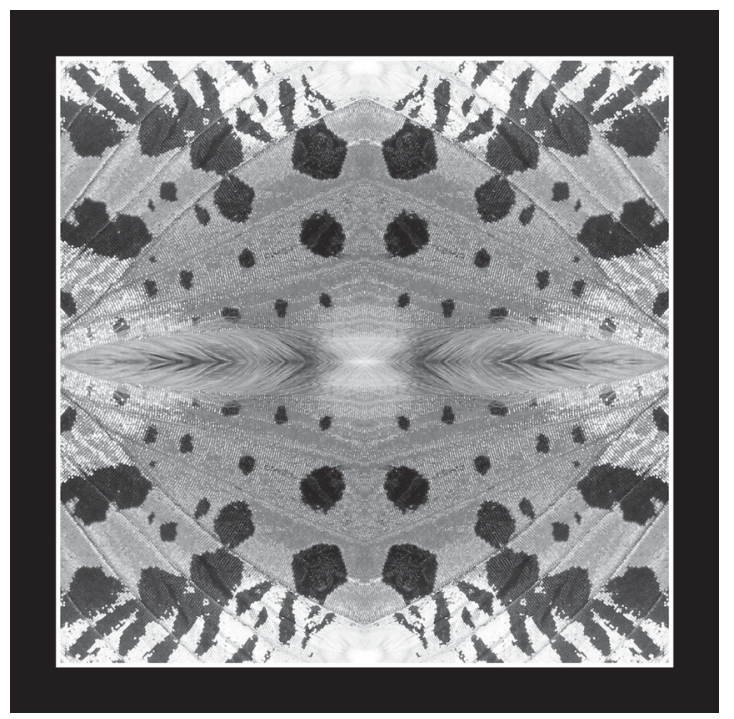

Urania Kaleidoscope-1st Place-Andrei Sourakov, Florida Museum of Natural History. "This image is created out of hindwings of Urania Sunset moth found in Madagascar. This moth is among the brightest of all Lepidoptera. This composition was meant to illustrate the beauty of moths that rivals beauty of any butterfly. It was also meant to emphasize the underlying symmetry found in the seemingly chaotic world."
At the conclusion of the submission deadline we had received 116 entries. We decided that online judging would be the easiest route. Our online judging process was accomplished through sending each judge an e-mail with both selection criteria (located under the guidelines section of the Web site) and a link to the Picasa album of entries. The judges were asked to review the images and associated statements then reply to the e-mail with an unranked list of their top ten images.

Once the first round of judging concluded, we tallied the results and were able to narrow it down to nine final images and a follow-up e-mail was sent to the judges. They were asked to send us a ranked list of their top five images. These results were assigned a point value (five points for first representation from the College of Engineering, College of Fine Arts, College of Liberal Arts and Sciences, and the Health Sciences colleges. As we progressed, it became clear that this process should have begun earlier in the timeline. Consequently, the panel consisted of only four judges:

- a UF Libraries' librarian,

- a retired emeritus professor of Chemistry (also a UF Libraries' donor and avid photographer),

- the dean of the College of Fine Arts, and

- a curator from the university's Museum of Natural History place, four points for second place, etc.) and tallied.

\section{Potential sponsors/partners}

In the spirit of forging relationships with other campus organizations and increasing funding for the contest, the idea of having partners and sponsors arose. We determined that we would approach organizations to be either a partner (this level would only be expected to provide publicity) or a sponsor (this level would be expected to contribute financially and provide publicity). For this year's contest we invited the Architecture and Fine Arts Library and the Health Science 
Center Libraries to partner with us because of the crossover appeal. In addition, we also extended an invitation to the UF Alumni Association.

\section{Reception planning}

The awards reception for the Elegance of Science took place in mid-February at the Marston Science Library. In the interest of promoting the welcoming environment of the library, the reception was on the main floor, open to the public, and no RSVP was required. The planning for this began in late January.

We designated a space that would be large enough to display the printed images on easels and project all of the entries on a large projection screen, as well as allowing for space for guests to mingle. A chemistry professor at the reception commented, "This is the first time I have been in the library in probably ten years. With electronic journals I haven't needed to."

\section{Challenges}

There were several challenges that arose during this process, most of which could be solved with an experienced party planner. The first challenge was time, specifically our underestimation of how much time we would need to devote to specific tasks. For example, because the judging process took a little longer than expected, we ended up with a very tight deadline to print the winning images. In addition, we did not make formal arrangements to have assistance for the reception preparation and consequently we were over committed on the day of the reception. Time devoted to food preparation will be significantly reduced due to university policies requiring catered food for all campus events.

As noted in a previous section, we did have some challenges involving resolution of the digital images submitted for the contest. There are still internal discussions about how this might be handled differently. A possible solution would be providing more specific guidance on our entry form.

A final challenge was lighting at the reception. We projected all the contestants' images using a portable LCD projector (with an 2000 lumen lamp) and a 80-inch projection screen. This made for a very nice set-up, however the fluorescent lighting in Marston is so bright that it hindered the vividness of the images significantly. We hoped that using a high-level LCD projector would eliminate this problem, but unfortunately it did not and there was no ability to dim/turn off a portion of the lights on the main floor. In the future, we will remove a portion of the light bulbs prior to the reception for ideal lighting levels.

\section{Future plans and development op- portunities}

It is our hope that the Elegance of Science contest will serve a secondary purpose as a development tool for Marston Library collection. The UF Libraries have experienced a flat budget causing us to make cuts to our collection.

In an effort to bolster to support our dwindling collections budget we, along with our associate dean for advancement and development, devised a plan to conduct an online auction of all the entries.

In the future, the permission agreement will be incorporated into the entry form. One printed copy of each entry would be sold and the proceeds would go an endowment for Marston. An additional endowment fund will also be put in place for donors who wish to contribute to the Elegance of Science contest itself. The goal will be to make the Elegance of Science annual contest and reception self-sustaining as well as being a support mechanism for the collections of Marston Science Library.

\section{Related readings}

Cornelia Dean, "She calls it 'phenomena.' everyone else calls it art," New York Times, June 12, 2007.

Howard Raskin, "Can an art gallery thrive in a science library? Communication through culture at Cornell University's Albert R. Mann Library," CERL News 70, no. 4 (2009): 226-9. $n$ 